\title{
Augmented Reality in Volumetric Medical IMAgING USING STEREOSCOPIC 3D Display
}

\author{
Sang-Moo Park ${ }^{1}$ and Jong-Hyo Kim ${ }^{1,2}$ \\ ${ }^{1}$ Biomedical Radiation Science, Graduate School of Convergence Science Technology, \\ Seoul National University, Korea \\ ${ }^{2}$ Associate Professor, Department of Radiology, Seoul National University, Korea
}

\begin{abstract}
This paper is written about augmented reality in medicine. Medical imaging equipment (CT, PET, MRI) are produced $3 D$ volumetric data, so using the stereoscopic $3 D$ display, observer feels depth perception. The major factors about depth-Convergence, Accommodation, Relative size are tested. Convergence and Accommodation have affected depth perception but relative size is negligible.
\end{abstract}

\section{KEYWORDS}

Augmented Reality, Volumetric, Convergence, Accommodation, Depth Perception, Stereoscopic.

\section{INTRODUCTION}

Augmented reality technology is applied in various fields and we consider applying to the technology under study. In particular, the application range of medical applications is various. [1 4] In the present medical Imaging equipment (CT, PER, MRI) are generating the volumetric Image. [5] However, the volumetric image can be observed using the current typical monitor (2Dmonitor). Display is one of the most important elements in the augmented reality technology. Volumetric image generated by the medical device is to provide the 3D image. If applying to the special display like that 3D display which provide to feel depth perception. We have a 3D image by applying a stereoscopic 3D display implementation left / right image generation during the experiment was based on the factors affecting the sense of depth.

\section{Prior Work}

We conducted a stereoscopic 3D related research. Many previous studies have been conducted regarding stereoscopic 3D. In the medical field, augmented reality implementation is ongoing. The study of the display has been in progress, which provides a virtual reality space to the viewers in order to realize the augmented reality. Stereoscopic 3D virtual reality research was conducted using the parallax barrier and HMD (Head Mount Display). [6, 7] Using the parallax barrier can be aware of the depth perception without special glasses. The parallax barrier technology, the location of the barrier in front of screen vertically divides the screen image to the left and right images are physically however parallax barrier technology have a problem, A fixed viewing position is required in order to feel the three-dimensional and the horizontal resolution of the Image is reduced. Stereoscopic cues provide improvement of depth perception in a very realistic way intensifying perception of surfaces and materials and also facilitating spatial localization. There were found with respect to the preceding findings stereoscopic factors 
International Journal of Computer Graphics \& Animat ion (IJCGA) Vol.5, No.2, April 2015 affecting 3D. The three components give a sense of depth are convergence of the eyes, Accommodation of the lens, The relative size $[8,9]$.

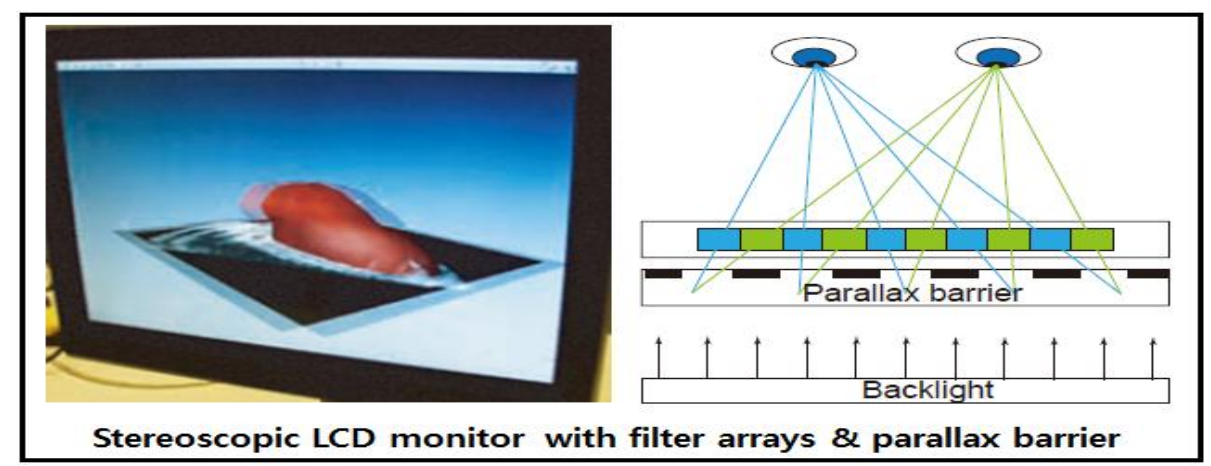

Figure 1.Parallax Barrier [10]

\subsection{Factors that affect the 3D stereoscopic}

\subsubsection{Convergence of the eyes}

Convergence is associated with both eyes movement when people look at an object. Depending on the distance of the object facing the difference between the both eyes of the movement takes place, Figure2 Convergence. The change in the both eyes angle occurs when looking at the far away of an object and the object when viewed a near. When looking at the object that is the difference between the distances of the binocular angle changes that occur in the brain of a person becomes recognize in depth.

\subsubsection{Accommodation of the lens}

The process by which the human eye changes optical power to maintain a clear image or focus on an object as its distance varies. Figure2 Accommodation when stare at the big white rectangleshaped pattern in a circle of black background, shows how small white rectangular pattern recognition do. The real pattern is clear both large and small rectangular pattern. However, focus on the large square pattern, small ones is blurred.

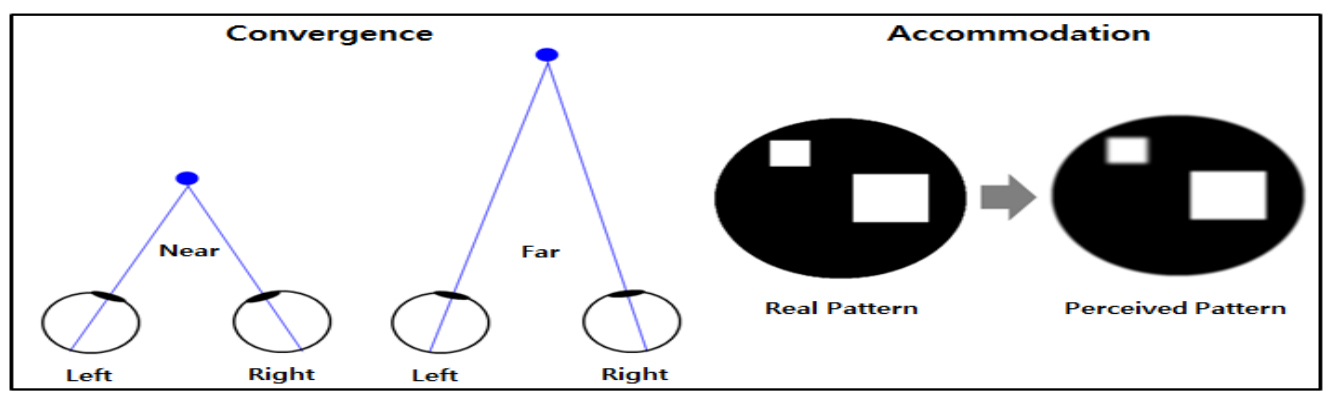

Figure 2. Convergence and Accommodation

\subsubsection{The Relate Size}

When a person is to recognize objects, the object size gives a sense of depth. 


\section{METHOD}

Our experiment was carried out with respect to the main factor mentioned above, the experiment was using 23inch 3D display, 3D glasses type is the active type (Shutter glasses), test pattern experiment was conducted on manufactured using Matlab. Test Pattern making was a fabrication method according to a side by side 3D video formats, which are implemented in 3D to change the 3D monitor Viewing distance is 700 [mm] was performed on the basis. Personnel involved in the experiment was carried out with ten men

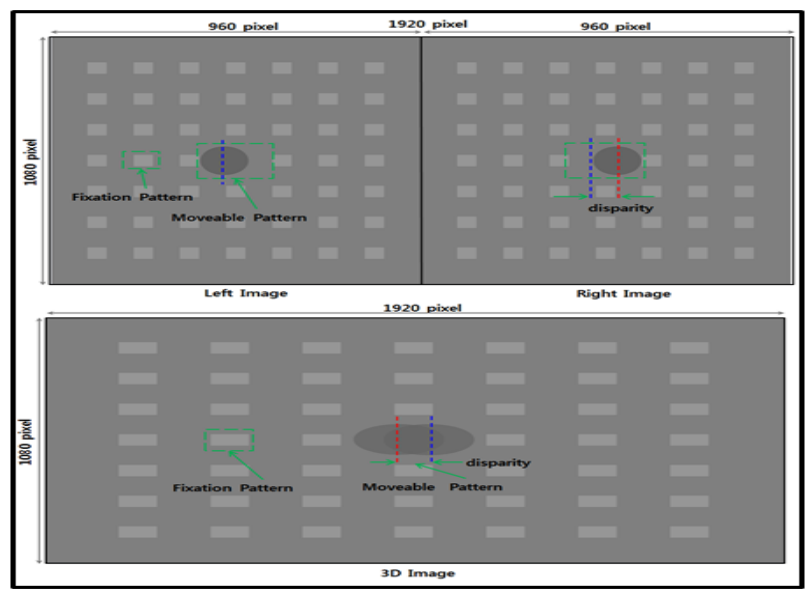

Figure 3. Test Pattern (Side by side format and 3D Image from monitor)

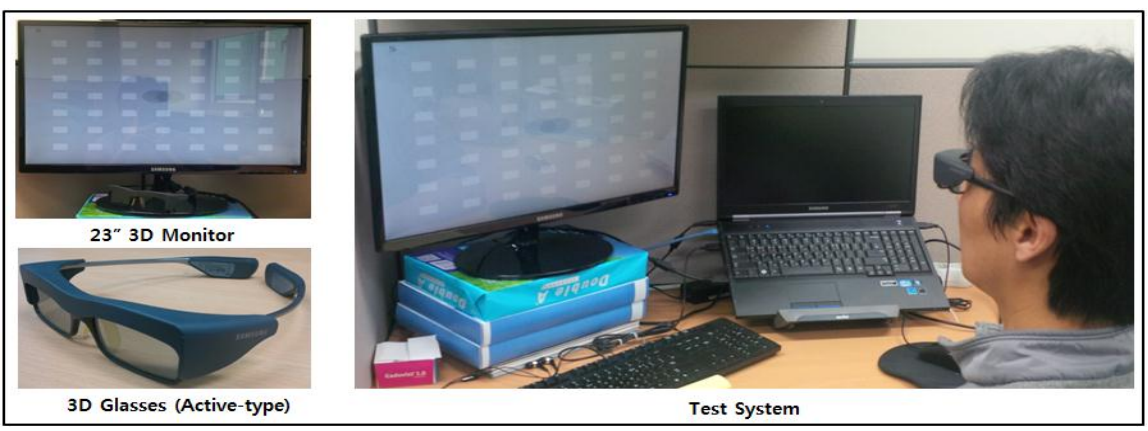

Figure 4. 23inch Stereoscopic 3D Monitor and Test System

\subsection{Convergence Change}

Test pattern was prepared under the conditions that change the object (moveable pattern) from behind to front into the display screen which was produced by controlling the disparity value of the left / right image. Depth of the pattern was made by computed based on the display pixel pitch and viewing distance. Limiting the depth was carried out in less than $700[\mathrm{~mm}]$, test pattern was the creation of 20 conditions in accordance with the disparity value. 
International Journal of Computer Graphics \& Animat ion (IJCGA) Vol.5, No.2, April 2015

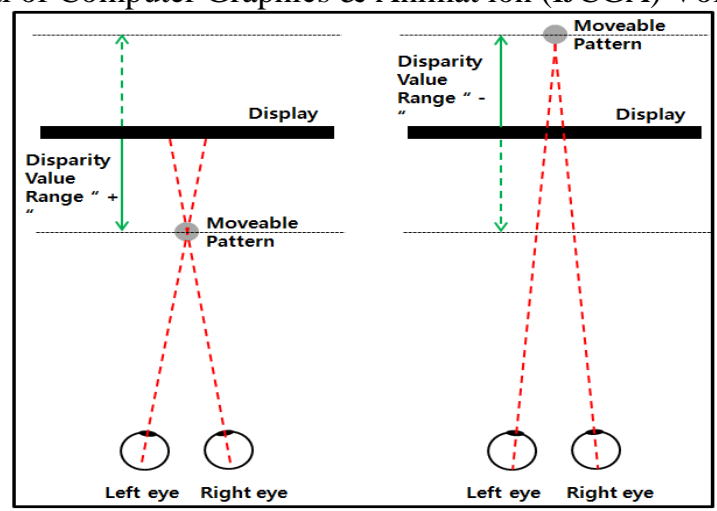

Figure 5. Change of the Convergence

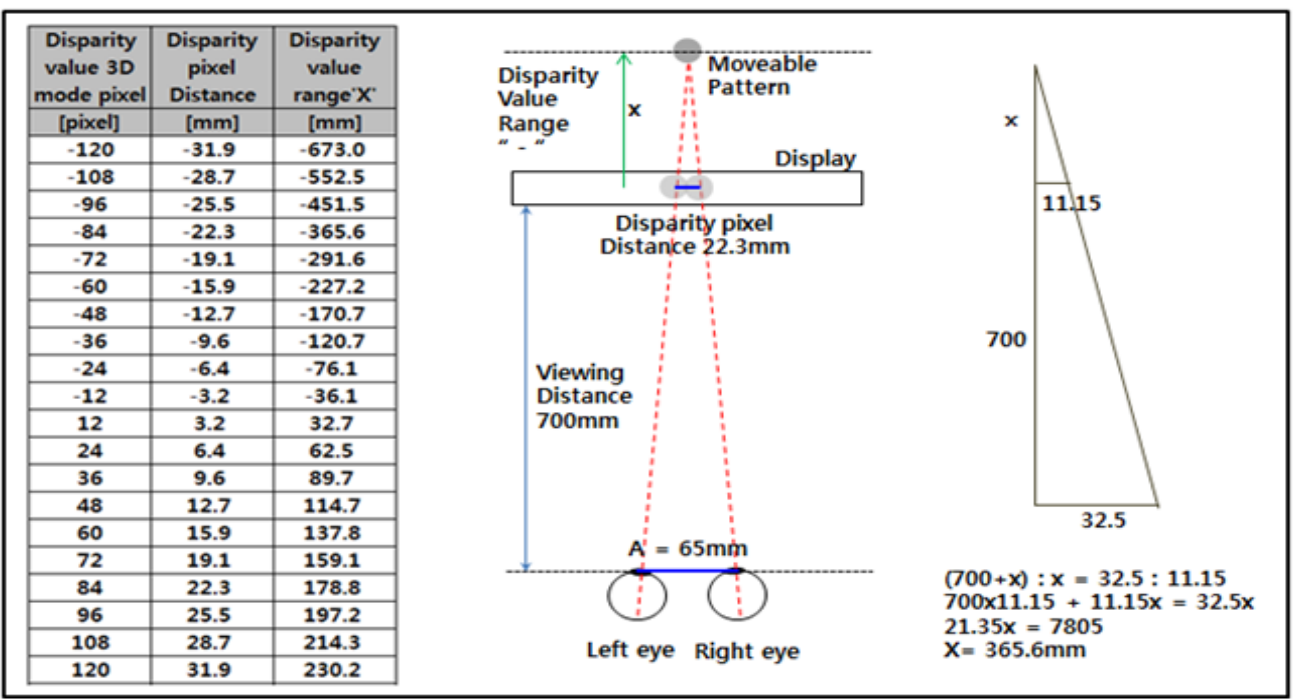

Figure 6. Test pattern condition and the calculation disparity value

\subsection{Accommodation}

Test pattern used in this experiment is in proportion to the distance of the 48 squares of the test pattern based on the middle of screen is different from the blurring effect was produced by. Blurring effect was used as a Gaussian filter the size of the filer mask was carried out in three different conditions $(3 \times 3,9 \times 9,15 \times 15$ pixels). Test pattern was the production of 10 pieces, depending on filter conditions.

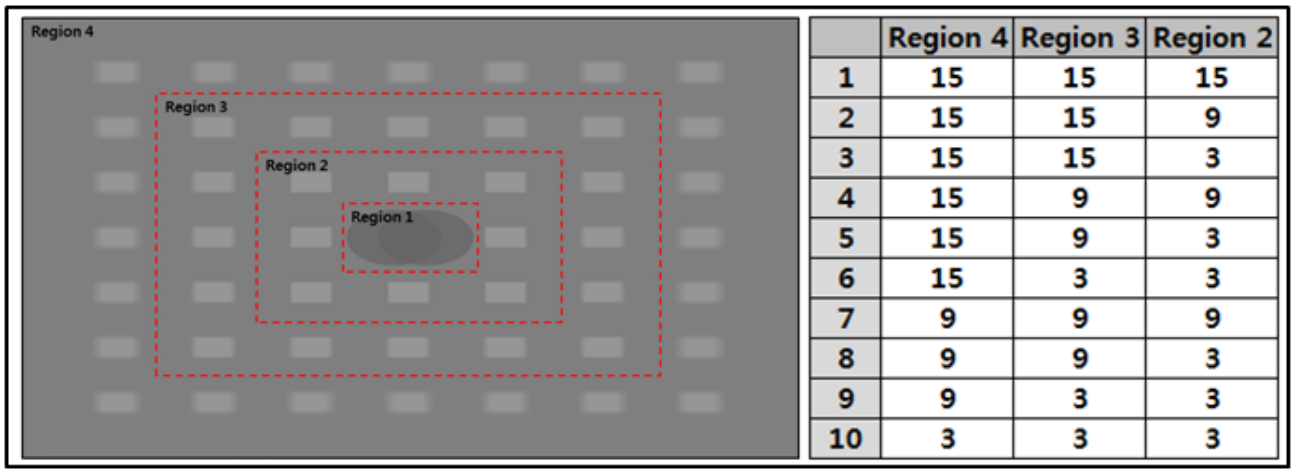

Figure 7. Accommodation Test Pattern and Experimental Table 
International Journal of Computer Graphics \& Animat ion (IJCGA) Vol.5, No.2, April 2015

\subsection{Relative Size}

Test pattern for checking the Relative size was produced from 13 Pattern changes the size of object (Circle), the area of interest to 50pxiels unit to 800pixels in 200pixels.

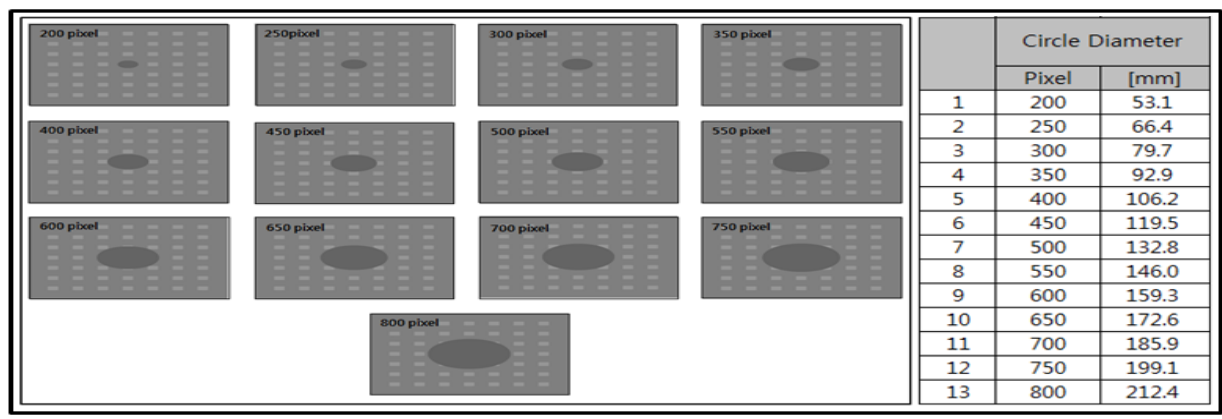

Figure 8. Relative Size Test Pattern and Pattern Size

\section{RESULT}

Convergence and Accommodation results appeared to affect the depth perception, however, that the relative size of effects depth perception is found to be insignificant. In case of the convergence, when the object is placed in front of the screen based on the depth perception appeared to be effective, but some observers have complained of dizziness when there is a case that the object is formed in front of the screen. The effective depth perception conditions are in front of the screen $138[\mathrm{~mm}]$ (disparity value 60) 159[mm] (disparity value 72).

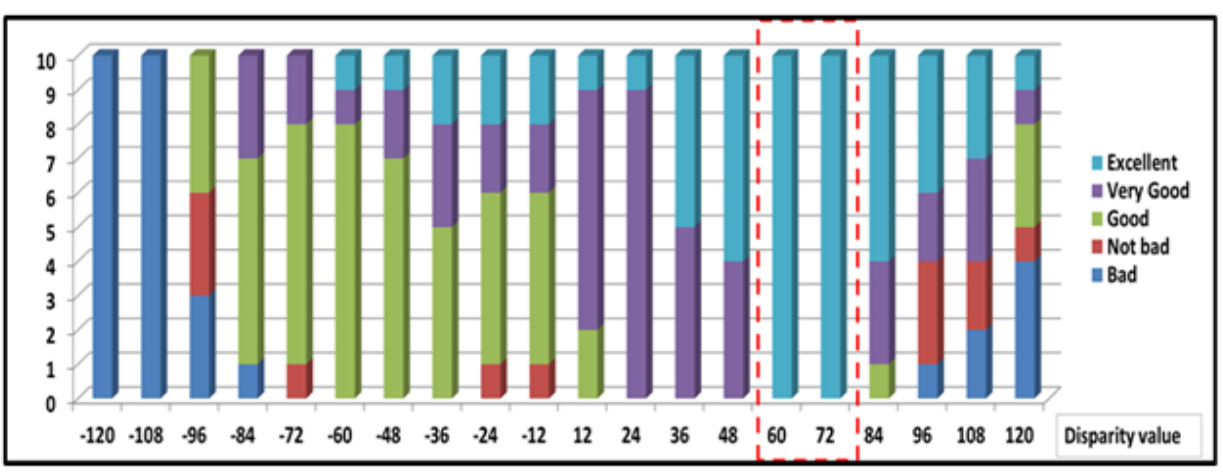

Figure 9. Convergence Test Results

Accommodation, experimental results showed that the number 5 condition is the most efficient for depth perception. The blurring effect applied sequentially in the middle of the screen based on the area showed that the region 2,3,4 applied mask size $3 \times 3,9 \times 9,15 \times 15$ pixels are the most effective in depth perception.

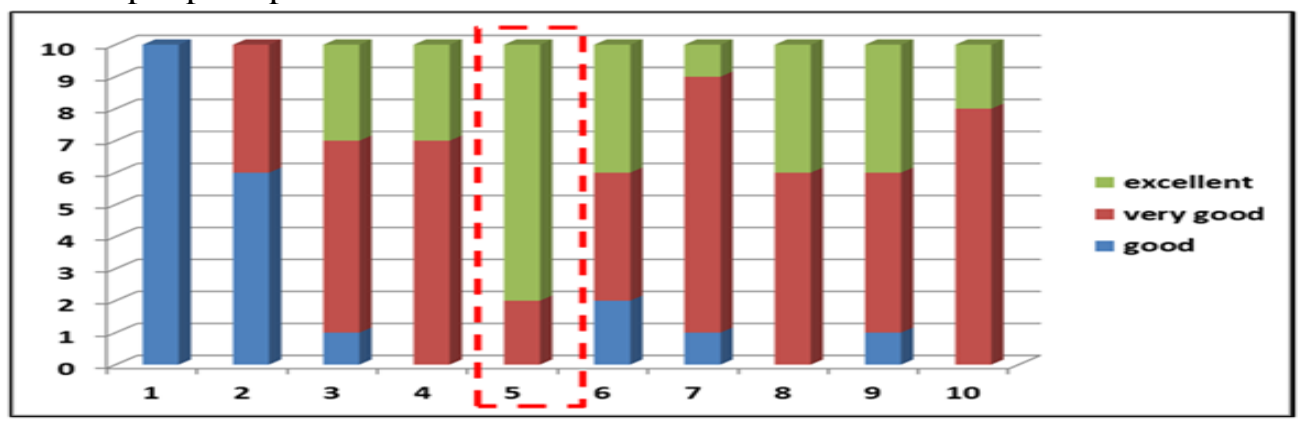

Figure 10. Accommodation Test Results 


\section{ConClusions}

International Journal of Computer Graphics \& Animat ion (IJCGA) Vol.5, No.2, April 2015

This paper shows that creating the stereoscopic 3D image from volumetric Medical Imaging, considering the display size and viewing distance should be create a stereoscopic image. The depth perception is formed in front of the screen than to form the behind has appeared to be more effective. Some people, however, that in front of the screen pattern for forming is to be noted that it can be complained of dizziness. And the blurring effect out of interest area human viewing was effective in the depth perception. However, Change in size of the object of interest was found to be effective that the depth perception is negligible. Future plans will proceed with the study of the relationship between awareness of things to control on-screen objects and been produced in stereoscopic 3D [11]

\section{REFERENCES}

[1] HELD, Robert T.; HUI, Tiffany T. A guide to stereoscopic 3D displays in medicine. Academic radiology, 2011, 18.8: 1035-1048.

[2] ZHAO, Yue, et al. 3D display technology in medical imaging field. In: Medical Imaging Physics and Engineering (ICMIPE), 2013 IEEE International Conference on. IEEE, 2013. p. 210-214.

[3] TREISMAN, Anne. Binocular rivalry and stereoscopic depth perception. Quarterly Journal of Experimental Psychology, 1962, 14.1: 23-37.

[4] KANG, Xin, et al. Stereoscopic augmented reality for laparoscopic surgery. Surgical endoscopy, 2014, 28.7: 2227-2235.

[5] SUETENS, Paul. Fundamentals of medical imaging. Cambridge university press, 2009. p.33-125

[6] SUETENS, Paul. Fundamentals of medical imaging. Cambridge university press, 2009. p.205-208

[7] SUETENS, Paul. Fundamentals of medical imaging. Cambridge university press, 2009. p.214-218

[8] COLE, Forrester, et al. Directing Gaze in 3D Models with Stylized Focus. Rendering Techniques, 2006, 2006: 17th.

[9] POYADE, Matthieu; REYES-LECUONA, Arcadio; VICIANA-ABAD, Raquel. Influence of Binocular Disparity in Depth Perception Mechanisms in Virtual Environments. In: New Trends on Human-Computer Interaction. Springer London, 2009. p. 13-22.

[10] SUETENS, Paul. Fundamentals of medical imaging. Cambridge university press, 2009. p.208, Figure 8.28

[11] AZARI, Hossein; CHENG, Irene; BASU, Anup. Stereo 3D mouse (S3D-Mouse): Measuring ground truth for medical data in a virtual 3D space. In: Engineering in Medicine and Biology Society, 2009. EMBC 2009. Annual International Conference of the IEEE. IEEE, 2009. p. 5744-5747.

\section{AUTHORS}

\section{Sang-Moo Park}

Received the B.S. from Chung-Ang University, Korea. in Electricity and Electronic Engineering.in 2001. From 2001 Feb to 2013 Aug working in Samsung Electronics (Display Processing Engineer). Form 2013 Sep , sturdy in Biomedical Radiation Science, Graduate School of Convergence Science Technology, Seoul National University, Suwon, Korea. 